\title{
Electronic properties and charge state of gold monomers and chains adsorbed on alumina thin films on $\mathrm{NiAl}(110)$
}

\author{
N. Nilius, ${ }^{1, *, \dagger}$ M. V. Ganduglia-Pirovano, ${ }^{2, *, \ddagger}$ V. Brázdová, ${ }^{3}$ M. Kulawik, ${ }^{1}$ J. Sauer, ${ }^{2}$ and H-J. Freund ${ }^{1}$ \\ ${ }^{1}$ Fritz-Haber-Institut der MPG, Faradayweg 4-6, Berlin, Germany \\ ${ }^{2}$ Institut für Chemie, Humboldt Universität zu Berlin, Brook-Taylor-Straße 2, Berlin, Germany \\ ${ }^{3}$ London Centre for Nanotechnology, University College London, 17-19 Gordon Street, London WC1H OAH, United Kingdom
}

(Received 11 September 2009; revised manuscript received 22 December 2009; published 21 January 2010)

\begin{abstract}
The adsorption of $\mathrm{Au}$ on a thin alumina film on $\mathrm{NiAl}(110)$ has been investigated with scanning tunneling microscopy (STM) and spectroscopy as well as density-functional theory (DFT). Gold monomers are identified as the main surface species at low coverage. According to the DFT, the adatoms are negatively charged and exhibit only a single occupied state in the alumina band gap. These results are compatible with the distinct bias-dependent contrast of Au monomers in the STM. With increasing exposure, Au chains of different length develop on the oxide surface. The adchains exhibit a number of maxima in conductance spectroscopy that are assigned with the help of DFT calculations to two sets of quantum well states with Au $6 s-d_{z 2}$ and Au $p$ character. By analyzing the node structure of the highest occupied states, the charge state of the adchains is determined. Chains containing three to seven $\mathrm{Au}$ atoms are found to host up to three excess electrons, in accordance with the charge-transfer model developed for Au adsorption on ultrathin oxide films.
\end{abstract}

DOI: 10.1103/PhysRevB.81.045422

PACS number(s): 73.21.Fg, 68.37.Ef, 68.47.Jn, 71.15.Mb

\section{INTRODUCTION}

The unusual growth behavior of metals, and in particular of gold, on thin oxide films has been subject to various experimental and theoretical studies in recent years. ${ }^{1,2}$ In contrast to the three-dimensional (3D) metal growth on most bulk oxides, the formation of one-dimensional (1D) (Refs. $3-5)$ and two-dimensional (2D) aggregates, ${ }^{6,7}$ has been revealed on ultrathin oxide films prepared on metal supports. A charge transfer from the metal substrate into the adsorbates was identified as the major driving force for the development of low-dimensional ad-structures. ${ }^{8,9}$ The charge exchange is triggered by a shift of the adsorbate affinity level below the Fermi level of the metal-oxide system and enables the activation of efficient charge-mediated interaction schemes between the adsorbate and the metal-oxide system. The negatively charged ad-species experiences a Coulomb attraction toward the oxide cations and stimulates a polaronic distortion of the oxide lattice below, increasing the adsorbateoxide binding strength. ${ }^{10}$

In an attempt to increase the charge transfer from the support, the ad-material maximizes the contact area with the oxide surface. In density-functional theory (DFT) calculations, charged 2D clusters were consequently found to be lower in energy than their neutral 3D counterparts, ${ }^{11,12}$ a result that was later verified experimentally for $\mathrm{Au}$ on $\mathrm{MgO}$ thin films. ${ }^{6}$ In the limit of ultrasmall clusters, even 1D cluster shapes emerge. In this configuration, the transfer electrons from the support can be distributed along the cluster axis, which strongly reduces the internal Coulomb repulsion of the ad-system. As a consequence, 1D cluster shapes become energetically favorable despite of their relatively small number of metal-metal bonds. The formation of 1D atomic chains has been observed experimentally for Au deposition on $\mathrm{MgO}$ and alumina thin films. ${ }^{4,5}$

This paper provides a detailed analysis of the electronic structure of charged $\mathrm{Au}$ atoms and chains that spontaneously develop on the alumina/NiAl(110) system. The alumina thin film belongs to the best-studied metal-oxide systems ${ }^{13,14}$ and a detailed structure model is available. ${ }^{15,16} \mathrm{Gold}$, on the other hand, is particularly interesting because of its outstanding catalytic performance as nanomaterial. ${ }^{17}$ The charged nature of $\mathrm{Au}$ chains on the alumina/NiAl film has already been demonstrated in an earlier scanning tunneling microscopy (STM)/DFT study ${ }^{4}$ however a comprehensive discussion of the electronic structure of the chains as a function of their length is still missing. Systems with linear geometry are ideally suited to examine electron confinement in one dimension, as the resulting quantum well states (QWS) display particularly simple symmetries. Such quantization effects have been analyzed for 1D systems on metal and semiconductor surfaces, for instance for $\mathrm{Au}, \mathrm{Pd}$, and $\mathrm{Cu}$ chains on $\mathrm{NiAl}(110)$ and $\mathrm{Cu}(111)$, respectively. ${ }^{18-20}$ By exploring spatial modulations in the electron density as a function of energy, the dispersion relation of those QWS has been reconstructed with the STM, although the method does usually not provide $k$-space information. In this study, the charge state of the oxide-supported chains is obtained as additional result from the observed quantization behavior. The particularly simple electronic structure of the 1D Au chains thus provides insight into the fundamental interaction mechanisms between $\mathrm{Au}$ and the metal-supported alumina film.

It should be noted at this point that electronic-structure data of individual metal clusters are usually not accessible to nonlocal spectroscopic techniques. The reason is the strong dependence of the cluster electronic properties on the exact number and arrangement of atoms in the system. ${ }^{21}$ Given the size and shape distribution of clusters in an ensemble, nonlocal experiments are always subject to substantial inhomogeneous broadening effects that generally circumvent the elucidation of single-cluster properties.

\section{EXPERIMENTAL AND THEORETICAL DETAILS}

The measurements were performed in an ultrahigh vacuum (UHV) STM operated at $4.5 \mathrm{~K} .{ }^{22}$ The sample elec- 
tronic structure was probed with differential conductance spectroscopy using lock-in technique at open feedback-loop conditions. ${ }^{23}$ The alumina film was prepared by oxidizing a clean $\mathrm{NiAl}(110)$ surface in $5 \times 10^{-7} \mathrm{mbar}_{2}$ at $580 \mathrm{~K}$, followed by heating in UHV to $1150 \mathrm{~K} \cdot{ }^{13,14}$ The procedure leads to the formation of an atomically flat, 5- $\AA$-thin oxide film that completely wets the metal surface. According to an earlier STM-DFT study, ${ }^{15}$ the film consists of four layers, which are from the bottom to the top: (i) an interfacial $\mathrm{Al}_{i}$ layer consisting of $\mathrm{Al}_{5}$ and $\mathrm{Al}_{7}$ rings, (ii) a hexagonal $\mathrm{O}_{i}$ layer being in registry with (iii) the $\mathrm{Al}_{s}$ surface layer, and finally (iv) an $\mathrm{O}_{s}$ surface layer. The oxide unit cell (17.86 $\times 10.55 \AA$ ) contains in total $16 \mathrm{Al}_{i}, 24 \mathrm{O}_{i}, 24 \mathrm{Al}_{s}$, and $28 \mathrm{O}_{s}$ atoms, bringing the stoichiometry to $\mathrm{Al}_{10} \mathrm{O}_{13}$. The film has a band gap of $\sim 6.4 \mathrm{eV}$ that separates the $\mathrm{O} 2 p$-derived valence band from the conduction band with Al $3 s$ character. The dominant film defects are dislocation lines between adjacent alumina domains, being introduced to reduce misfit-induced strain with the NiAl support. ${ }^{14,15}$ Single $\mathrm{Au}$ atoms were evaporated onto the freshly prepared film at $10 \mathrm{~K}$ from a high-purity Au wire wrapped around a tungsten filament. Due to their initial thermal energy, the incoming $\mathrm{Au}$ atoms are able to perform a transient diffusion into their equilibrium binding configurations.

Spin-polarized DFT calculations are carried out with the gradient-corrected Perdew-Wang 91 exchange and correlation functional and a plane-wave basis set (energy cutoff 250 $\mathrm{eV})$, as implemented in the VASP code. ${ }^{24}$ The Al $(3 s, 3 p)$, Ni $(3 d, 4 s), \mathrm{O}(2 s, 2 p)$, and $\mathrm{Au}(5 d, 6 s)$ electrons were treated as valence states. The core electrons were described by the projector-augmented-wave method. ${ }^{25}$ The Brillouin zone is sampled at the $\Gamma$ point. The computational cell covers two alumina unit cells $\left(8 \mathrm{Al}_{10} \mathrm{O}_{13} \cdot 66 \mathrm{NiAl}\right)$. Due to the incommensurability between oxide and $\mathrm{NiAl}$ lattice constants along the [001] direction, the oxide unit cell is slightly expanded in this direction to enforce lattice matching. ${ }^{15}$ Gradient corrected functionals as used here are known to underestimate the band gaps of insulators and the calculated orbitals are therefore systematically lower in energy than their experimental counterparts.

\section{RESULTS AND DISCUSSION}

\section{A. Gold monomers and dimers}

Single $\mathrm{Au}$ atoms appear as circular features of $6-7 \AA$ diameter mainly on the defect-free terraces of the alumina film [Fig. 1(a)]. A preferential Au attachment to the oxide domain boundaries is not revealed. The adatoms exhibit a distinct bias-dependent contrast [Fig. 1(b)]. They are imaged as 1- $\AA$-high protrusions at negative sample bias (occupied states) but decrease their apparent height at positive bias and even turn into shallow depressions above $+3.0 \mathrm{~V}$. Simultaneously, their shape transforms from Gaussian maxima at negative bias to protrusions surrounded by a dark ring (sombrero shape) around $+2.5 \mathrm{~V}$ and simple holes above $+3.0 \mathrm{~V}$ (Fig. 2). Besides the Au monomers, also larger ad-species with circular or slightly elliptical shapes are observed on the alumina film (Fig. 1). Their apparent height is less bias dependent and changes from $1 \AA$ at negative bias to $3 \AA$ at
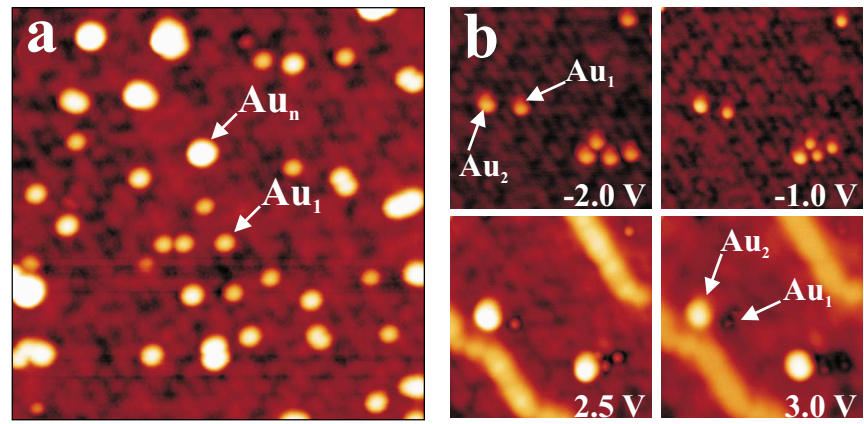

FIG. 1. (Color online) (a) STM topographic image of $0.05 \mathrm{ML}$ $\mathrm{Au}$ on alumina/NiAl (110) $\left(18 \times 18 \mathrm{~nm}^{2},-1.0 \mathrm{~V}\right)$. (b) Bias series of a surface region containing $\mathrm{Au}$ monomers and dimers (15 $\times 15 \mathrm{~nm}^{2}$ ). Bright lines emerging above $2.5 \mathrm{~V}$ are domain boundaries in the oxide film. Note the strong variation in the Au appearance at different bias voltages.

+2.5 V. These larger ad-species never turn negative in the accessible bias window of the experiment (Fig. 2) and are assigned to $\mathrm{Au}$ dimers.

Apart from the topographic contrast, the different adspecies can be identified via their $d I / d V$ fingerprint in conductance spectroscopy [Fig. 3(a)]. The spectra of Au monomers show a monotonously increasing $d I / d V$ signal without distinct maxima and are rather similar to the alumina background spectra. The absence of Au electronic states between the Fermi level $\left(E_{F}\right)$ and the alumina conduction band is compatible with the continuously decreasing height of the adatoms in topographic images (Figs. 1 and 2). Dimers, on the other hand, exhibit a pronounced $d I / d V$ peak at around $+2.6 \mathrm{~V}$ that is responsible for their bright appearance at this bias. The peak position slightly varies due to different binding configurations of the dimers on the alumina surface. At negative bias, no $d I / d V$ peaks are observable for monomers and dimers, although a finite topographic height above the alumina film suggests the availability of Au-induced states for electron transport at negative bias.
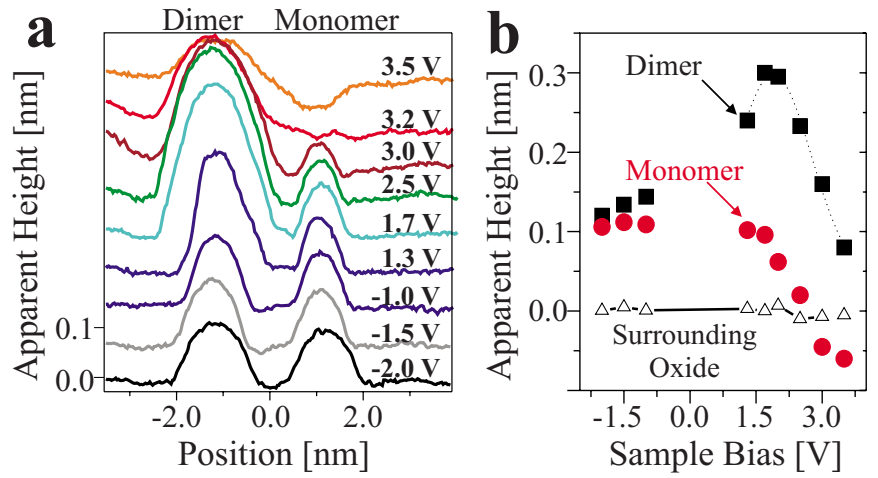

FIG. 2. (Color online) (a) Height profiles along a line that contains an $\mathrm{Au}$ monomer and a dimer on alumina/NiAl(110) measured for different sample voltages. Profiles are offset for clarity. (b) Plot of their apparent height as a function of the imaging bias. The triangles depict the height of the oxide film measured in $10 \AA$ distance from a monomer. Both monomer and surrounding oxide are imaged with negative contrast around the conduction-band onset of alumina $(3.0 \mathrm{eV})$ due to the absence of states for electron transport. 

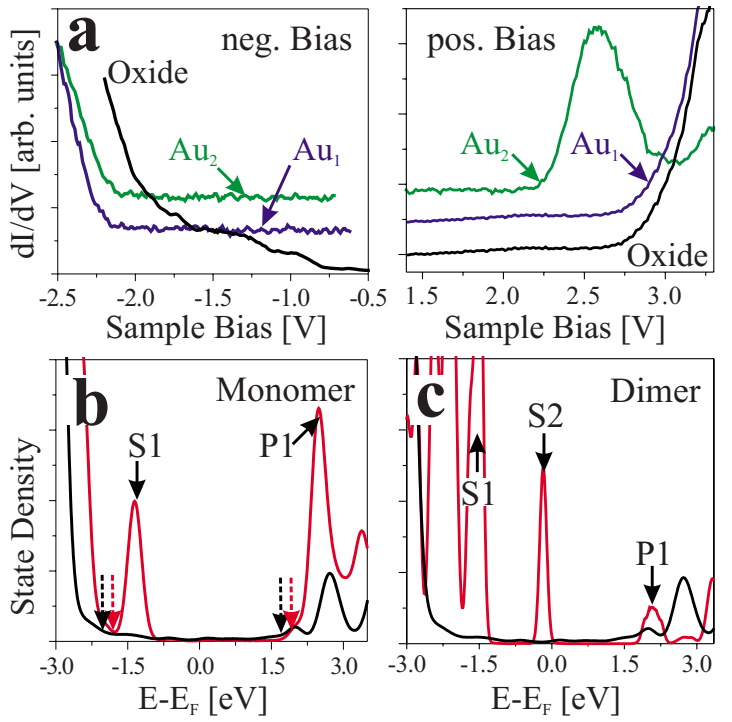

FIG. 3. (Color online) (a) Filled-state (set point: $-2.5 \mathrm{~V}$ ) and empty-state (set point: $+3.0 \mathrm{~V}) d I / d V$ spectra of an Au monomer, a dimer, and the bare alumina film. The spectra are offset for clarity. Only the dimer shows a resonance at $+2.6 \mathrm{~V}$. (b) Calculated total DOS of the clean alumina (black) and of the $\mathrm{Au}_{1}$-alumina system (red) projected onto the $\mathrm{Al}-\mathrm{O}_{3}$ unit in the oxide surface layer below the adsorption site. The onsets of the alumina bands are marked with broken arrows in both cases. (c) Total DOS of the $\mathrm{Au}_{2}$-alumina system projected onto the gold dimer (red). Black curve as in (b).

The experimental signature can be compared to DFT calculations for the different Au ad-species. As demonstrated in an earlier study, ${ }^{4} \mathrm{Au}$ atoms preferentially bind to the surface $\mathrm{Al}_{s}$ and not to the $\mathrm{O}_{s}$ atoms in the film. Upon bond formation, the $\mathrm{Al}_{s}$ is lifted above the surface plane and the bond to the $\mathrm{O}_{i}$ ion in the layer below breaks. This bond rupture is accompanied by an electron transfer from the $\mathrm{Al}_{s}$ to the $\mathrm{Au}$, while the subjacent $\mathrm{O}_{i}$ is reduced. Particularly stable binding configurations are now obtained when this $\mathrm{O}_{i}$ species is able to restore its charge state by forming a bond with an $\mathrm{Al}$ atom in the $\mathrm{NiAl}$ support. In this case, Au-binding energies between 1.5 and $2.0 \mathrm{eV}$ are revealed, while this value drops below $1.4 \mathrm{eV}$ without NiAl participation. Gold adsorption to the alumina film is therefore governed by such "special" $\mathrm{Al}_{s}$ sites that enable a substrate-mediated bonding scheme due to their location above an $\mathrm{Al}$ atom in the NiAl surface.

The electronic structure of $\mathrm{Au}$ monomers bound to special $\mathrm{Al}_{s}$ sites comprises an occupied $s-d_{z 2}$ state at $-1.3 \mathrm{eV}$ below $E_{F}$ (highest occupied molecular orbital, HOMO) and an unoccupied state with $p_{\perp}$ character at $+2.5 \mathrm{eV}$ (lowest unoccupied molecular orbital, LUMO) [Fig. 3(b), S1 and P1]. The HOMO derives from the Au $6 s$ orbital that is half filled in gas-phase $\mathrm{Au}$ and becomes doubly occupied on the oxide surface due to the charge transfer from the metal support. In dimers, the $s-d_{z 2}$ orbitals of the two Au atoms hybridize and form new states at -0.3 and $-1.5 \mathrm{eV}$ [Fig. 3(c), S1 and $\mathrm{S} 2] .{ }^{26}$ Both states are filled, which brings the total number of $s$ electrons to four and the nominal charge state of the dimer to $-2|e|$. The charge transfer is compatible with the rupture of two oxide $\mathrm{Al}_{s}-\mathrm{O}_{i}$ bonds below the dimer. The LUMO at $+2.0 \mathrm{~V}$ is again of $p_{\perp}$ character and has down-shifted with respect to the monomer into the alumina band gap [Fig, 3(c), P1].

From the frontier orbitals of the monomer and dimer, only the dimer LUMO shows up in the $d I / d V$ spectra as a peak at $2.6 \mathrm{~V}$ [Fig. 3(a)]. The LUMO of the $\mathrm{Au}_{1}$ is not detected due to its location inside the alumina conduction band that entirely dominates the electron transport into the sample. Also, the occupied gold states are not revealed for two possible reasons. (i) Filled orbitals are usually confined between the ad-species and the support in accordance with their binding character and have little overlap with the tip wave functions. (ii) Tunneling out of occupied Au states might be associated with a temporal charging of the ad-species as the extracted HOMO electron needs to be replenished via electron tunneling through the alumina film. Such charging effects might induce an energy barrier that cannot be overcome during spectroscopy.

So far, the excess charges on the Au species on alumina/ $\mathrm{NiAl}(110)$ thin films have only been derived from the DFT calculations. However, STM imaging and spectroscopy can provide some evidence for the charged nature of $\mathrm{Au}$ as well. A first hint comes from the distinct sombrero shape of $\mathrm{Au}$ monomers in STM images taken between +2.0 and $+3.0 \mathrm{~V}$ [Fig. 1(b)]. Sombrero shapes are considered as fingerprint for charged adsorbates on insulating and semiconducting supports and have been observed around charged impurities on $\mathrm{Si}(111),{ }^{27} \mathrm{NaCl},{ }^{28}$ and $\mathrm{TiO}_{2}(110)$ surfaces. ${ }^{29}$ The sombrero shape reflects the local distortion of the host bands in vicinity to the point charge. In the case of thin insulating films on metal supports, the effect is enhahced by Friedel oscillations of the metal electrons around the charge perturbation.

A second indication comes from the distinct contrast reversal of the Au monomers in images taken above $3.0 \mathrm{~V}$ (Fig. 2). Imaging with negative contrast implies that the available state density for tunneling into the adsorbate is lower than into the unperturbed oxide film. ${ }^{23}$ This suggests that the monomer does not have electronic states by its own for electron transport around $3.0 \mathrm{~V}$, in agreement with the calculated position of its LUMO inside the oxide conduction band. However, the adatom seems even to remove state density from the oxide patch underneath at this particular energy. This behavior can be rationalized by a local up-shift of the alumina conduction band in presence of the negatively charged adsorbate. Whereas in the unperturbed film, new states for electron transport become available when the bias voltage matches the band onset at $\sim 3.0 \mathrm{~V},{ }^{30}$ this threshold is not reached around the adatom. Consequently, the tip still probes the low state density of the oxide band gap and the adatom is imaged with negative contrast with respect to the surrounding oxide. The up-shift of the oxide states is in part explained by a local distortion of the alumina band structure due to the Al-O bond cleavage. Additionally, it results from the electrostatic repulsion exerted by the anionic $\mathrm{Au}$ onto adjacent alumina states that shift to higher energies. A positively charged impurity, in contrast, would lower the band onset of the oxide film, which is not observed here. ${ }^{27}$ The latter effect becomes however evident around the dislocation lines of the alumina film that are positively charged due to an electron transfer out of confined defect levels into the $\mathrm{NiAl}$ support. ${ }^{16,30}$ The associated down-shift of the oxide conduc- 


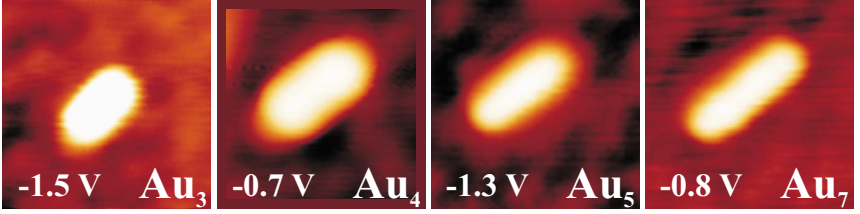

FIG. 4. (Color online) Self-assembled Au chains containing 3-7 atoms on alumina/ $\mathrm{NiAl}(110)\left(5 \times 5 \mathrm{~nm}^{2}\right)$.

tion band renders the lines defects bright in STM images taken close to the conduction-band onset [see Fig. 1(b)].

The local modification of the alumina bands in vicinity to the $\mathrm{Au}$ adatom is reproduced in the DFT calculations. Apart from the two Au-induced states, marked S1 and P1 in Fig. 3(b), the onset of the valence and conduction bands has moved to higher energy (see arrows). According to the $d I / d V$ spectra, none of the Au-induced states directly contributes to the tunneling transport due to their high spatial localization. The up-shift of the conduction band and the concomitant reduction of the state density can however be probed by the STM and dictates the negative contrast of monomers in images taken at $\sim 3.0 \mathrm{~V}$. A similar contrast reversal is not observed for Au dimers, although they even host two extra electrons. The reason is that a removal of state density at the conduction-band onset is counterbalanced by the induction of the $\mathrm{Au}_{2}$-LUMO [see P1 in Fig. 3(c)], which enables efficient electron transport through the dimer and gives rise to its bright appearance.

\section{B. Linear gold clusters}

At higher metal exposure, one-dimensional Au aggregates with different length and a unique orientation along the $\mathrm{NiAl}[001]$ orientation develop on the alumina film (Fig. 4).
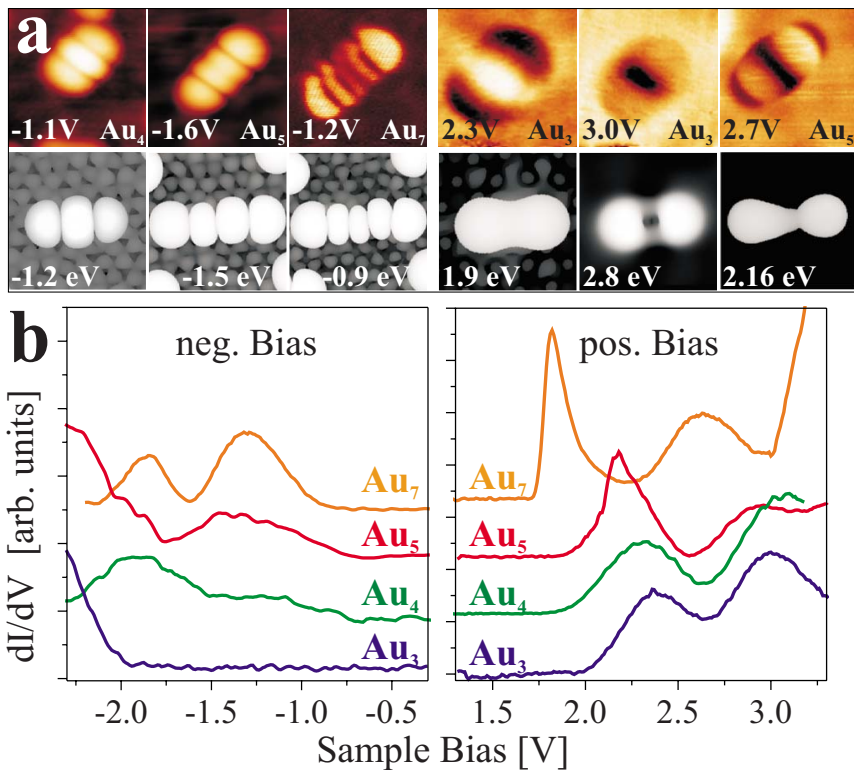

FIG. 5. (Color online) (a) Conductance maps taken at the position of the HOMO of $\mathrm{Au}_{4}, \mathrm{Au}_{5}$, and $\mathrm{Au}_{7}$ (left) and the LUMO of $\mathrm{Au}_{3}$ and the LUMO+1 of $\mathrm{Au}_{3}$ and $\mathrm{Au}_{5}$ (right, $5 \times 5 \mathrm{~nm}^{2}$ ). The calculated orbital shapes are shown for comparison. (b) Filled-state (set point: $-2.0 \mathrm{~V}$ ) and empty-state (set point: $+3.0 \mathrm{~V}) \mathrm{dI} / \mathrm{dV}$ spectra of the respective Au adchains. Spectra are offset for clarity.
The shortest chain is $8 \AA$ long and assigned to a linear trimer. The longest one (length: $22 \AA$ ) contains seven atoms, as suggested by DFT simulations. ${ }^{4}$ The evolution of the electronic structure of the chains as function of their length is probed with $d I / d V$ spectroscopy and summarized in Fig. 5. With the exception of the trimer, all adchains exhibit conductance peaks above and below $E_{F}$. Filled-state spectroscopy is apparently feasible for bigger clusters, most likely due to the larger number and better delocalization of electrons in the potential well that facilitates electron extraction. Whereas a continuous redshift of the leading $d I / d V$ peak with increasing chain length is revealed from the empty-state spectra, no clear trend is observed for the filled states. For selected $d I / d V$ maxima, the symmetry of the underlying orbitals could be deduced from conductance images taken at the respective peak energies. The HOMO symmetry is always governed by a regular sequence of lobes along the cluster axis, whereby the lobe number is characteristic for adchains of a given length [Fig. 5(a): left]. The LUMO and LUMO+1, on the other hand, display only one or two maxima [Fig. 5(a): right]. The shapes of unfilled orbitals are also subject to certain variations even if the length and configuration of the chains seem to be identical. A complete $d I / d V$ series is presented for an $\mathrm{Au}_{7}$ chain in Fig. 6. In this particular example, the HOMO and HOMO-1 are characterized by five and four
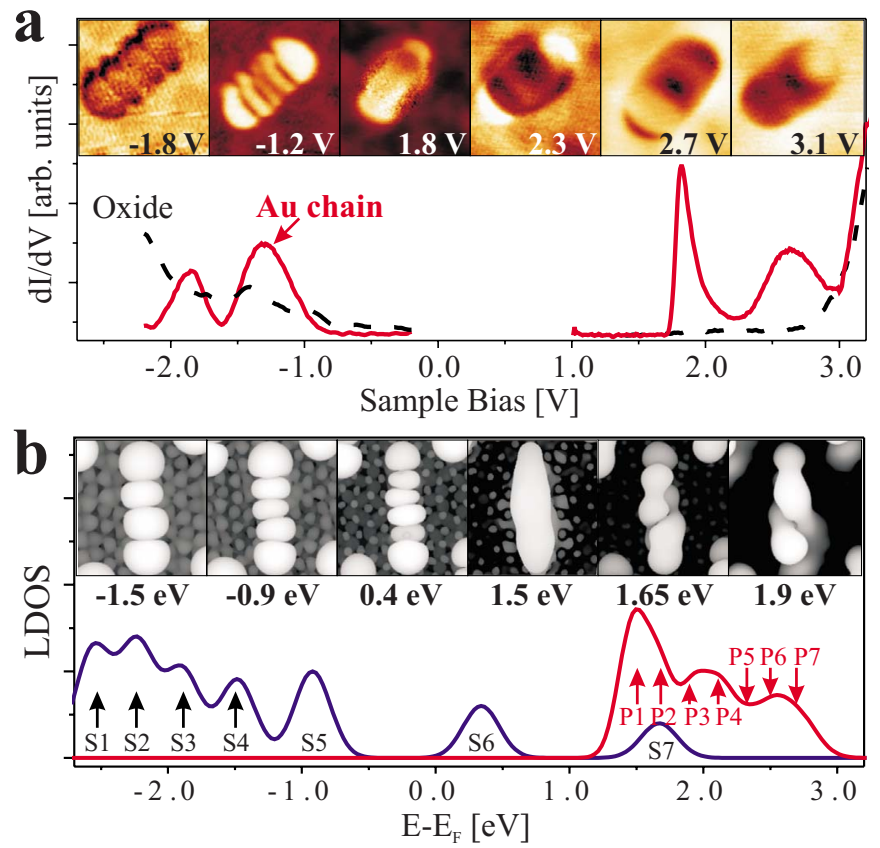

FIG. 6. (Color online) (a) Conductance spectra of an $\mathrm{Au}_{7}$ chain and the bare oxide film. Insets display $d I / d V$ maps taken at the peak positions of the respective spectrum $\left(5 \times 5 \mathrm{~nm}^{2}\right)$. The region around the Fermi level could not be accessed in spectroscopy because tip stabilization at low bias values triggers desorption of the $\mathrm{Au}$ adstructures. (b) Calculated LDOS for an $\mathrm{Au}_{7}$ chain. Blue line denotes the seven Au $s-d_{z 2}$-like QWS (termed S1-S7), while the red line depicts the $p_{\perp}$ state density. The quantized nature of the $p_{\perp}$ states is not resolved due to the small energy separation between adjacent levels. Their energy positions are however marked by arrows (P1-P7). The calculated orbital shapes for the fourth to sixth $s$-like and the first to third $p_{\perp}$-like QWS are shown as insets. 


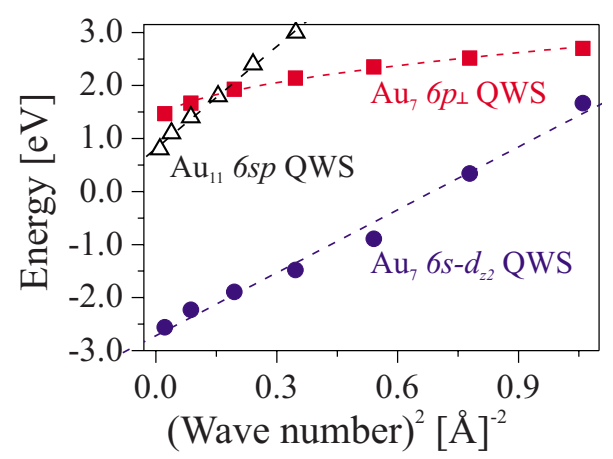

FIG. 7. (Color online) Dispersion relation shown as a function of the squared wave number $(k=n \pi / L)^{2}$ for the $s-d_{z 2}$ (blue circles) and the $p_{\perp}$ QWS (red squares) in an $\mathrm{Au}_{7}$ chain on alumina/ $\mathrm{NiAl}(110)$. Black triangles depict the dispersion of the $6 s p$-derived QWS in an $\mathrm{Au}_{11}$ chain prepared on bare $\mathrm{NiAl}(110)$ for comparison (from Ref. 18). For the $s$-derived QWS, the dispersion relation is parabolic $\left(E \propto k^{2}\right)$, while a direct $E \propto k$ proportionality is revealed for the $p_{\perp}$-derived levels (broken lines).

lobes along the axis, respectively. The unfilled chain orbitals are less defined; the somewhat blurred LUMO exhibits no nodal plane, while several, asymmetric maxima are observed for the higher states.

The computed electronic structure of the Au chains is essentially described by the interplay of two size-dependent trends. First, the $s-d_{z 2}$-like HOMO of the Au monomer splits into a set of $m$ partly occupied states with strong $s$ character in an $m$-atom chain. These states are marked with $\mathrm{S} 1-\mathrm{S} 7$ in Fig. 6. Second, $m$ empty states with $p$ character develop upon chain formation from the $p_{\perp}$-like LUMO of the single atom. The $s$-like state manifold exhibits the characteristic properties of free-electron-like QWS confined in a linear box potential with infinite walls. ${ }^{18}$ The eigenfunctions of such a potential are sinusoidals $\left(\psi_{n}=\sin k_{n} x\right)$ described by a quantum number $n$ and a wave-number $k_{n}$ that is proportional to the inverse box length $(k=n \pi / L){ }^{31}$ The resulting electrondensity distribution $\psi_{n}^{2}$ in each QWS is given by $n$ maxima separated by $n-1$ nodes along the chain axis. Such orbital shapes are indeed observed for the occupied orbitals of the Au chains, both experimentally and theoretically (Figs. 5 and 6). Another indication for the free-electron character of the $s$-like QWS comes from their dispersion relation. The computed energies of the seven QWS in an $\mathrm{Au}_{7}$ chain follow the expected parabolic dependence on the quantum number $n$ according to ${ }^{31} E_{n}=E_{0}+\frac{\hbar^{2}}{2 m_{e f f}}\left(\frac{\pi n}{L}\right)^{2}$. The two fit parameters of the model dispersion are the potential depth $E_{0}=-2.65 \mathrm{eV}$ and the effective electron mass $m_{\text {eff }}=0.85 m_{e}$ (Fig. 7). The unoccupied QWS show a nearly linear dispersion $\left(E_{0}\right.$ $=1.3 \mathrm{eV}$, slope $1.4 \mathrm{eV} \AA$ ), indicating that the particle-inthe-box model is not applicable to the $p_{\perp}$-like QWS. Instead, a harmonic potential might be used, as it accounts for the flatting of the potential when approaching the vacuum energy and is even compatible with the observed linear dispersion relation. ${ }^{31}$ The box potential for the low-lying $s$ states and the harmonic potential for the empty $p_{\perp}$ states are thus the two limiting cases to describe the real electron potential in the Au chains.

Closer inspection of the diagram in Fig. 7 reveals that the $s$-like state manifold of the $\mathrm{Au}_{7}$ chain is more than half filled, as five eigenstates are below and only two above the Fermi level. Compared to a neutral $\mathrm{Au}_{7}$ chain with three filled, three unfilled, and one half-filled QWS (reflecting the intermixing of seven half-filled Au $6 s$ orbitals), the ad-chain is hence threefold negatively charged. Two additional results support this conclusion. (i) Both the experimental and the computed $\mathrm{HOMO}$ of the $\mathrm{Au}_{7}$ chain exhibit five lobes along the chain axis, identifying it as the fifth $s$-like QWS (Fig. 6). Assuming double occupancy of all states below $E_{F}$, the chain carries ten $s$ electrons in total, three more than a neutral chain with a half-filled $s$ band. (ii) The DFT-Bader charge for an $\mathrm{Au}_{7}$ on the alumina thin film amounts to $-2.88|e|$, in good agreement with the charge state deduced from the band diagram. Additionally, the DFT calculations find three broken $\mathrm{Al}-\mathrm{O}$ bonds below the heptamer chain, being indicative for three transferred electrons. A negative charging is also revealed for the shorter adchains, with the pentamer chain containing three and the tetramer and trimer holding two extra electrons. ${ }^{4}$ It should be noted that the electron transfer from the $\mathrm{NiAl}$ into the $\mathrm{Au}$ ad-species is in perfect agreement with the charge-mediated interaction of Au with other oxide films. ${ }^{5-9}$ It satisfies not only the electronegative character of gold, but additionally enables effective electrostatic and polaronic bonding schemes with the oxide surface. ${ }^{8}$

Based on the electronic structure discussed above, the $d I / d V$ spectra of the differently sized Au chains are now easily interpreted. The $d I / d V$ peaks observed at negative bias are assigned to the $s$-like QWS, whereby the quantum number $n$ of the respective level is directly given by the number of electron-density maxima along the chain axis. The HOMOs of $\mathrm{Au}_{4}$ and $\mathrm{Au}_{5}$ are therefore identified as the third and fourth $s$-like eigenstates in the linear potential, while the HOMO and HOMO- 1 of $\mathrm{Au}_{7}$ are attributed to the fifth and fourth QWS, respectively. Among the large number of experimental adclusters, not a single one featured the computed properties of an $\mathrm{Au}_{6}$ chain. The absence of this particular species might be related to the odd number of valence electrons in the system (six intrinsic plus three transfer electrons) and the occurrence of a singly occupied QWS at the Fermi level. The $\mathrm{Au}_{6}$ chain has therefore a magnetic ground state that renders this chain configuration energetically unfavorable. Note that the linear Au trimer is magnetic as well, but this configuration is frequently found on the surface. The linear $\mathrm{Au}_{3}$ has however a lower energy than all the nonlinear configurations, whereas several linear and nonlinear $\mathrm{Au}_{6}$ isomers exist with comparable energies. ${ }^{4}$

The correlation between theory and experiment is less perfect for the unoccupied states of the different Au chains. The trimer LUMO at $2.3 \mathrm{~V}$ exhibits a single intensity maximum in the chain center, similar to the lowest $p_{\perp}$-like QWS found in DFT (Fig. 5). For the LUMO+1, a single nodal plane is visible in the $d I / d V$ image, identifying it as second $p_{\perp}$-like QWS. Similar conclusions are drawn for the $\mathrm{Au}_{5}$ chain (Fig. 5). The Au heptamer exhibits even three unoccupied states [Fig. 6(a)], however only the first one at $1.8 \mathrm{~V}$ can be directly assigned to the lowest $p_{\perp}$ QWS due to its homogenous $d I / d V$ intensity distribution. ${ }^{32}$ The higher states observed at 2.7 and $3.1 \mathrm{~V}$ are of low symmetry and an unambiguous correlation to QWS in an ideal potential well is not possible. However, also the computed energy levels are in- 
trinsically asymmetric [Fig. 6(b)]. One obvious reason for the distorted orbital shapes is the small energy separation between adjacent $p_{\perp}$-like QWS, which leads to an overlap of states with different symmetry. In addition, also an unoccupied $s-d_{z 2}$ QWS emerges in the energy window of the $p_{\perp}$ states, corrupting their symmetry. Finally, the electron potential along the chain axis is not homogenous as Au adsorption takes place at differently coordinated $\mathrm{Al}_{s}$ atoms and the binding contribution of the NiAl support exhibits spatial variations. ${ }^{4}$ As a consequence, the binding energy of the chain atoms varies by more than $1 \mathrm{eV}$, which in turn affects the delocalization of the $p_{\perp}$-like wave functions. The occupied $s$-like QWS are less disturbed by the inhomogeneous chain properties due to their pronounced free-electron character. The potential modulations along the chain axis cannot be accounted for with the computational cell used for the calculations due to the incommensurate nature of the NiAlalumina system and the associated shifts in the interfacial registry.

It is finally instructive to compare the electronic properties of Au chains grown on alumina/ $\mathrm{NiAl}(110)$ to the ones of similar adchains prepared on a metal support. As both quantum systems have a comparable morphology, deviations in their electronic properties might shed light into the particular influence of the support material. Gold chains containing up to 20 atoms were assembled on $\mathrm{NiAl}(110)$ via atom manipulation with the STM tip. ${ }^{18}$ These chains exhibit a set of unoccupied QWS with pronounced 6sp character and a parabolic dispersion relation. ${ }^{33}$ A second set of filled $s$ - $d$ QWS, starting at $-4 \mathrm{eV}$ below $E_{F}$, has been identified by DFT, but could not be observed experimentally due to a strong overlap with the NiAl states. The electronic structure of NiAlsupported adchains therefore arises from two contributions: (i) the $\mathrm{Au} 6 s$ orbital of the adatom hybridizes with the $\mathrm{NiAl} s p$ band and splits into a filled $s-d$ and an empty $s p$ resonance. ${ }^{34}$ The parity of the splitting with respect to $E_{F}$ ensures charge neutrality of the adatom on the metal surface. (ii) Both states develop into a parabolic band when Au atoms are assembled into a linear chain. ${ }^{18,33}$ The $s p$ band above the Fermi level is hereby characterized by a small effective mass of $m_{\text {eff }}=0.5 \mathrm{~m}_{\mathrm{e}}$, suggesting a strong coupling between neighboring chain atoms and a high degree of electron delocalization. The interatomic coupling originates from the direct overlap of the $\mathrm{Au}$ atomic orbitals, but has a substantial substrate-mediated contribution as well. On the alumina film, the Au QWS are of distinctively different character (Fig. 7).
The unoccupied band is dominated by the intermixing of Au $6 p$ orbitals, while the filled states below $E_{F}$ arise almost exclusively from the $\mathrm{Au} 6 s$ orbitals with little $d$ intermixing. The coupling between adjacent chain atoms is much smaller in this case, as substrate-mediated interactions are essentially absent on the insulating oxide film. Consequently, the effective electron mass of the $s$-like QWS is almost 2 times larger for oxide compared to metal-supported chains, although the interatomic distance is even $10 \%$ shorter on the alumina film.

\section{CONCLUSION}

The electronic properties of $\mathrm{Au}$ adatoms and linear clusters have been analyzed on alumina thin films by a combined STM and DFT approach. Negative charging is revealed for all Au-related adstructures and results from an electron transfer through the insulating film. For $\mathrm{Au}$ monomers, experimental evidence for the charged nature comes from a distinct contrast reversal at positive bias, which is traced back to an up-shift of the oxide bands around the anionic gold. For higher metal exposures, the development of linear Au clusters is observed on the alumina film, the electronic structure of which is dominated by two overlapping systems of QWS. The lower one is of $s-d_{z 2}$ character and partly filled, while the upper one is completely empty and has $p_{\perp}$ character. Both sets of QWS are detectable with conductance spectroscopy, offering the unique possibility to characterize the valence electronic structure of a well-defined linear quantum system. From comparing the electron occupancy of $s$-like QWS in the Au adchains to an idealized neutral system, the charge state of the clusters on the oxide support is determined. In agreement with a Bader analysis, accumulation of one to three excess electrons is revealed for clusters containing up to seven atoms. Our study demonstrates how the structural and electronic properties of nanosystems can be analyzed even on insulating supports by the rigorous application of STM and DFT as investigative tools.

\section{ACKNOWLEDGMENTS}

J.S. and H.J.F. acknowledge support from the Cluster of Excellence "Unifying Concepts in Catalysis" funded by the DFG as well as from the "Fond der Chemischen Industrie." The calculations were carried out at the supercomputer system of The North-German Supercomputing Alliance (Norddeutscher Verbund zur Förderung des Hoch- und Höchstleistungsrechnens, HLRN).

\footnotetext{
*Corresponding author.

†nilius@fhi-berlin.mpg.de

${ }^{\ddagger}$ Present address: Institute of Catalysis and Petroleochemistry, Campus UAM, Cantoblanco, 28049 Madrid, Spain; vgp@chemie.huberlin.de

${ }^{1}$ T. Risse, S. Shaikhutdinov, N. Nilius, M. Sterrer, and H.-J. Freund, Acc. Chem. Res. 41, 949 (2008); H.-J. Freund and G. Pacchioni, Chem. Soc. Rev. 37, 2224 (2008).
}

${ }^{2}$ D. W. Goodman, J. Catal. 216, 213 (2003).

${ }^{3}$ M. Kulawik, N. Nilius, and H.-J. Freund, Phys. Rev. Lett. 96, 036103 (2006).

${ }^{4}$ N. Nilius, M. V. Ganduglia-Pirovano, V. Brázdová, M. Kulawik, J. Sauer, and H.-J. Freund, Phys. Rev. Lett. 100, 096802 (2008).

${ }^{5}$ V. Simic-Milosevic, M. Heyde, X. Lin, T. König, H.-P. Rust, M. Sterrer, T. Risse, N. Nilius, H.-J. Freund, L. Giordano, and G. Pacchioni, Phys. Rev. B 78, 235429 (2008). 
${ }^{6}$ M. Sterrer, T. Risse, M. Heyde, H.-P. Rust, and H.-J. Freund, Phys. Rev. Lett. 98, 206103 (2007).

${ }^{7}$ X. Lin, N. Nilius, H.-J. Freund, M. Walter, P. Frondelius, K. Honkala, and H. Häkkinen, Phys. Rev. Lett. 102, 206801 (2009).

${ }^{8}$ G. Pacchioni, L. Giordano, and M. Baistrocchi, Phys. Rev. Lett. 94, 226104 (2005).

${ }^{9}$ P. Frondelius, H. Häkkinen, and K. Honkala, New J. Phys. 9, 339 (2007).

${ }^{10}$ L. Giordano, U. Martinez, S. Sicolo, and G. Pacchioni, J. Chem. Phys. 127, 144713 (2007).

${ }^{11}$ D. Ricci, A. Bongiorno, G. Pacchioni, and U. Landman, Phys. Rev. Lett. 97, 036106 (2006).

${ }^{12}$ M. Walter, P. Frondelius, K. Honkala, and H. Häkkinen, Phys. Rev. Lett. 99, 096102 (2007).

${ }^{13}$ M. Bäumer and H.-J. Freund, Prog. Surf. Sci. 61, 127 (1999).

${ }^{14}$ M. Kulawik, N. Nilius, H.-P. Rust, and H.-J. Freund, Phys. Rev. Lett. 91, 256101 (2003).

${ }^{15}$ G. Kresse, M. Schmid, E. Napetschnig, M. Shishkin, L. Köhler, and P. Varga, Science 308, 1440 (2005).

${ }^{16}$ M. Schmid, M. Shishkin, G. Kresse, E. Napetschnig, P. Varga, M. Kulawik, N. Nilius, H.-P. Rust, and H.-J. Freund, Phys. Rev. Lett. 97, 046101 (2006).

${ }^{17}$ M. Haruta, T. Kobayashi, H. Sano, and N. Yamada, Chem. Lett. 16, 405 (1987); M. Haruta, CATTECH 6, 102 (2002).

${ }^{18}$ N. Nilius, T. M. Wallis, and W. Ho, Science 297, 1853 (2002); J. Phys. Chem. B 109, 20657 (2005).

${ }^{19}$ S. Fölsch, P. Hyldgaard, R. Koch, and K. H. Ploog, Phys. Rev. Lett. 92, 056803 (2004).

${ }^{20}$ N. Oncel, A. van Houselt, J. Huijben, A. S. Hallbäck, O. Gurlu, H. J. W. Zandvliet, and B. Poelsema, Phys. Rev. Lett. 95, 116801 (2005).
${ }^{21}$ Clusters of Atoms and Molecules, edited by H. Haberland (Springer, Berlin, 1994); Metal Clusters, edited by W. Ekardt (Wiley, Chichester, 1999).

${ }^{22}$ H.-P. Rust, J. Buisset, E. K. Schweizer, and L. Cramer, Rev. Sci. Instrum. 68, 129 (1997).

${ }^{23}$ J. Tersoff and D. R. Hamann, Phys. Rev. Lett. 50, 1998 (1983).

${ }^{24}$ J. P. Perdew, J. A. Chevary, S. H. Vosko, K. A. Jackson, M. R. Pederson, D. J. Singh, and C. Fiolhais, Phys. Rev. B 46, 6671 (1992); G. Kresse and J. Furthmüller, Comput. Mater. Sci. 6, 15 (1996).

${ }^{25}$ P. E. Blöchl, Phys. Rev. B 50, 17953 (1994); G. Kresse and D. Joubert, ibid. 59, 1758 1999).

${ }^{26}$ The $z$ direction corresponds to the $\mathrm{Au}-\mathrm{Au}$ bond axis.

${ }^{27}$ R. J. Hamers, J. Vac. Sci. Technol. B 6, 1462 (1988).

${ }^{28}$ J. Repp, G. Meyer, F. E. Olson, and M. Persson, Science 305, 493 (2004).

${ }^{29}$ M. Batzill, K. Katsiev, D. J. Gaspar, and U. Diebold, Phys. Rev. B 66, 235401 (2002).

${ }^{30}$ N. Nilius, M. Kulawik, H.-P. Rust, H.-J. Freund, Phys. Rev. B 69, 121401(R) (2004).

${ }^{31}$ C. Kittel, Introduction to Solid State Physics (Wiley, New York, 1996).

${ }^{32}$ The small width and asymmetric shape of the $\mathrm{Au}_{7}$ LUMO gives room for another explanation. Here, the state corresponds to an initially occupied $s$-like QWS that shifts above the Fermi level in the tip-electric field and reappears in the spectra at elevated positive bias. See for details S. W. Wu, G. V. Nazin, X. Chen, X. H. Qui, and W. Ho, Phys. Rev. Lett. 93, 236802 (2004).

${ }^{33}$ M. Persson, Phys. Rev. B 72, 081404(R) (2005).

${ }^{34}$ N. Nilius, T. M. Wallis, M. Persson, and W. Ho, Phys. Rev. Lett. 90, 196103 (2003). 